\title{
A themed journal issue on deprescribing
}

\author{
Nina Barnett, ${ }^{1}$ Barry Jubraj ${ }^{2,3}$
}

We are delighted to present this themed issue on 'deprescribing', a subject that has gained increasing importance in prescribing practice across the world over the last decade. For example, at a recent European General Practitioner conference that attracted over 3000 participants, four symposia were devoted to medication appropriateness, polypharmacy and deprescribing. The appetite for safe deprescribing was clear, but clinicians (we consider pharmacists to be clinicians) voiced concerns including a lack of robust guidance, patient attitudes, and a fear of medicolegal consequences from deprescribing. Our themed issue provides an introduction to the deprescribing debate, with articles ranging from reviews by international experts on the current problems surrounding deprescribing, to a description of practical tools to support the process. We have also attempted to broaden the deprescribing debate by including articles on the patient view of deprescribing, a legal perspective, and the role of nurses working with patients, pharmacists and doctors to make deprescribing decisions collaboratively. Our themed issue also considers deprescribing in special populations such as older people with falls, patients in care homes, and, more briefly, in paediatrics and people with learning disabilities. In short, we hope to provide the reader with an accessible, practical overview of the emerging area of deprescribing.

In the first of our review articles, Polly Duncan and colleagues outline key steps in the deprescribing process (see page 37 ) and identify that while the harms of inappropriate polypharmacy are established, there is a paucity of evidence around the benefit of deprescribing in general practice settings. Focusing on the UK perspective, the authors suggest that doctors in general practice are well placed to undertake medication reviews to address polypharmacy and undertake deprescribing. In

\footnotetext{
${ }^{1}$ Medicines Use and Safety Division, NHS Specialist Pharmacy Service, England and London North West Healthcare NHS Trust, Middx, Harrow, UK; ${ }^{2}$ Institute of Pharmaceutical Sciences, King's College London, London, UK; ${ }^{3} \mathrm{NIHR}$ CLAHRC for Northwest London

Correspondence to Barry Jubraj, Institute of Pharmaceutical Sciences, King's College London, 150 Stamford Street, London SE1 9NH, UK; barry.jubraj@ kcl.ac.uk
}

their article, Shane Cullinan and colleagues from University College, Cork remind us that, despite deprescribing in older, multimorbid patients being desirable, multiple prescribers, interprofessional relationships, patient perspectives, and lack of evidence contribute to the ongoing deprescribing challenge (see page 43). The authors suggest that the identification of appropriate instances for deprescribing and supporting decisions will contribute to improved practice. We also have a thought-provoking, deliberately challenging article in which Professor Doron Garfinkel invites readers to think critically about their own perspectives on polypharmacy and to consider when a multidrug approach to deprescribing may be in the patients' best interests (see page 16). He describes his rationale for deprescribing a number of medicines at one time and encourages us to consider when a serial, slow deprescribing process may not be the ideal option for patients.

Professor Ian Scott considers the variety of guidelines and tools to support deprescribing, some with evidence of effectiveness and others supported by a theoretical approach (see page 51). While most approaches surveyed achieve reductions in the number of inappropriate medicines, the style of tools and the level of guidance varies from principles-based to drugspecific, and we are reminded that there is no single approved guideline or tool; and that more evaluation is required. In the absence of a validated tool, Alan Cassels and colleagues take readers on a journey that led to the creation and dissemination of an innovative tool to support deprescribing (see page 3). With the use of an evidence-based approach and a Delphi-style methodology, a practical online tool has been made available and publicised through social media, including an entertaining YouTube video which provides a tuneful review of the benefits of deprescribing. The tool is currently the subject of a validation trial.

We include information on the progress of the SIMPATHY (Stimulating Innovation Management of Polypharmacy and Adherence in The Elderly) Project (see page 5). Funded through the European Union Health Programme 2014-2020, with 10 institutions from eight European countries participating, SIMPATHY has supported an extensive literature review to gather guidelines together. In addition, the project has compiled nine case studies showcasing six polypharmacy management processes and has analysed three processes occurring in the absence of polypharmacy programmes in other countries.

In a new perspective on deprescribing, we present an article co-written by a pharmacist who subsequently trained as a lawyer, outlining potential fears around deprescribing from a UK legal perspective (see page 21). The paper reassures readers that deprescribing requires the same care and diligence that clinicians exercise when prescribing, in order to act within the law.

Sam Barnett Cormack helps us to consider the elements of a consultation that a patient may want from their clinicians (see page 34). As a lay person with multiple conditions, he adds a patient perspective on managing multimorbidity and multiple medications as part of everyday life. We feel privileged to include his reflections in our special issue and invite readers to pay attention to his insights. Anne Cole and Heidi Wright also remind us that, when managed carefully, polypharmacy can be both appropriate and helpful (see page 70). They also emphasise the importance of collaborative decision making between clinicians and patients to ensure that deprescribing is an agreed process. To support the imperative of collaborative deprescribing, Lelly Oboh presents a case that demonstrates flexibility and skilled clinical behaviours, where the balance of evidencebased prescribing, managing risk and supporting the patient's desire for independence led to a negotiation with a mutually acceptable outcome (see page 58).

We continue our special issue with a number of articles that demonstrate a multidisciplinary approach to deprescribing. Johannes Ros and colleagues describe the impact of joint consultation between a clinical pharmacist and a clinical geriatrician to improve inappropriate prescribing for elderly patients in the hospital setting (see page 26). Vanessa Marvin and colleagues describe the contribution of pharmacists to falls-reduction-focused medication review towards reduced morbidity and increased quality of life (see page 10).

With an emphasis on the importance of the nursing role, Corina Naughton and Nicky Hayes highlight the importance of the position of nurses in observing the difficulty, medication burden and side effects when administering medicines, and the opportunity this offers to foster effective communication around polypharmacy (see page 47). Their article introduces the concept of 'nurse deprescribers', particularly for nurse prescribers to consider. In their article about successful medication 


\section{Editorial}

review and deprescribing in nursing homes, Wasim Baqir and colleagues helpfully suggest that the profession of the person who undertakes a medication review is less important than their ability, experience and the use of an accepted method for review (see page 30).

Finally, a small number of letters and cases that highlight the opportunity for deprescribing in specific populations are included. A junior pharmacist, Rukeya Begum, identifies the paucity of published literature on deprescribing in children, along with a consultant paediatric pharmacist, Stephen Tomlin (see page 70). They describe the context of the lack of evidencebased prescribing in children and how, despite this, polypharmacy can occur. Danielle Adams presents a case describing the well-publicised challenge of psychotropic use in an adult patient with learning disability (see page 69). Danielle also shares with us an engaging case that reminds readers of the impact that medication side effects can have on things that really matter to patients.

We are grateful for the opportunity to have guest-edited this themed issue on deprescribing. What has motivated us is the need to educate-not only 'top down' by producing a themed issue such as this, but also 'bottom up' ${ }^{1}$ in order to introduce the challenges of polypharmacy and deprescribing to healthcare students and novice practitioners. The progress made in London on this is summarised in a short article by us and Alan Poots (see page 7 ). We hope that these ideas and this themed issue will stimulate our readers to consider how to get patients and staff thinking practically about deprescribing.

EAHP Statement 6: Education and Research
Disclaimer This article presents independent research commissioned by the NIHR under the CLAHRC programme for NW London. The views expressed in this publication are those of the authors and not necessarily those of the NHS, NIHR or the Department of Health.

Competing interests None declared.

Provenance and peer review Commissioned; internally peer reviewed.

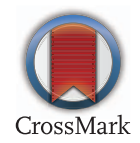

To cite Barnett N, Jubraj B. Eur J Hosp Pharm 2017;24:1-2.

Eur J Hosp Pharm 2017;24:1-2.

doi:10.1136/ejhpharm-2016-001118

\section{REFERENCE}

1 Jubraj B, Marvin V, Poots AJ, et al. A pilot survey of junior doctors' attitudes and awareness around medication review: time to change our educational approach? Eur J Hosp Pharm 2015;22:243-8. 